\title{
A Modern C++ Parallel Task Programming Library
}

\author{
Chun-Xun Lin* \\ ECE Dept, UIUC, IL \\ clin99@illinois.edu
}

\author{
Tsung-Wei Huang* \\ ECE Dept, University of \\ Utah, UT \\ twh760812@gmail.com
}

\author{
Guannan Guo \\ ECE Dept, UIUC, IL \\ gguo4@illinois.edu
}

\author{
Martin D. F. Wong \\ ECE Dept, UIUC, IL \\ mdfwong@illinois.edu
}

\begin{abstract}
In this paper we present Cpp-Taskflow, a $\mathrm{C}++$ parallel programming library that enables users to quickly develop parallel applications using the task dependency graph model. Developers formulate their application as a task dependency graph and CppTaskflow will manage the task execution and concurrency control. The task graph model is expressive and composable. It can express both regular and irregular parallel patterns, and developers can quickly compose large programs from small parallel modules. Cpp-Taskflow has an intuitive and unified API set. Users only need to learn the APIs to build and dispatch a task graph and no complex parallel programming concept is required. We have conducted experiments using both micro-benchmarks and realworld applications and Cpp-Taskflow outperforms state-of-the-art parallel programming libraries in both runtime and coding effort. Cpp-Taskflow is open-source and has been used in both industry and academic projects. From our users' feedback, we believe CppTaskflow can benefit the industry and research community greatly through its ease-of-programming and inspire new research directions in multimedia system/software design.
\end{abstract}

\section{KEYWORDS}

Parallel programming; task parallelism; task dependency graph

\section{ACM Reference Format:}

Chun-Xun Lin, Tsung-Wei Huang, Guannan Guo, and Martin D. F. Wong. 2019. A Modern C++ Parallel Task Programming Library. In Proceedings of the 27th ACM International Conference on Multimedia (MM '19), October 21-25, 2019, Nice, France. ACM, New York, NY, USA, 4 pages. https://doi.org/10.1145/3343031.3350537

\section{INTRODUCTION}

Multicore processors are prevalent from desktops, laptops, tablets to mobile devices. How to effectively utilize those computing resources to maximize software performance? This is a critical question that software developers must consider, especially when building complex parallel applications such as artificial intelligence, numerical simulation, machine learning and multimedia big data analytics [1] [2] [3] [4] [5]. Writing parallel code is considered much

\footnotetext{
*Both authors contributed equally to this research.

Permission to make digital or hard copies of all or part of this work for personal or classroom use is granted without fee provided that copies are not made or distributed for profit or commercial advantage and that copies bear this notice and the full citation on the first page. Copyrights for components of this work owned by others than ACM must be honored. Abstracting with credit is permitted. To copy otherwise, or republish, to post on servers or to redistribute to lists, requires prior specific permission and/or a fee. Request permissions from permissions@acm.org.

MM '19, October 21-25, 2019, Nice, France

(C) 2019 Association for Computing Machinery.

ACM ISBN 978-1-4503-6889-6/19/10 . \$15.00

https://doi.org/10.1145/3343031.3350537
}

more difficult than the sequential counterpart. Programmers have to pay extra attentions to the concurrency control to avoid unexpected behavior during runtime, for example, using locks to protect shared data or atomic variables to avoid data race. The situation is getting more challenging when applications exhibit complex data or operation dependency, which is typical in real-world problems. As a result, it's necessary to have an efficient approach to write parallel code.

In this paper, we present Cpp-Taskflow, a modern $\mathrm{C}++$ taskbased parallel programming library. Cpp-Taskflow was motivated from a real-world project of VLSI timing analysis [6]. CppTaskflow lets users express their parallelism using the intuitive task graph model. The task graph model is simple yet very powerful as it can represent both regular and irregular parallel patterns. The task graph model abstracts away complex concurrency management and allows users to focus on exploiting parallelism within their applications. Cpp-Taskflow provides well-designed APIs to keep the code concise and readable. We have a unified task graph construction interface for both static and dynamic parallelism, so users can learn those APIs quickly and utilize them to implement various parallel patterns. Cpp-Taskflow supports visualization for program debugging and profiling. Users can dump the task graph to inspect the program execution flow and they can view the thread activities in a Chrome browser. We have conducted experiments on a set of micro-benchmarks and a real-world application [7] against Intel Threading Building Blocks [8] and OpenMP [9]. CppTaskflow achieves comparable performance with fewer lines of code, faster runtime, and better scalability.

We understand each library has its own uniqueness and value, and it's up to users to decide which best suits their needs. Cpp-Taskflow has been used in many industrial and academic projects [10]. We are committed to free sharing of our technical innovation to facilitate the research in parallel computing, machine learning, and multimedia. We are working actively with our users to improve Cpp-Taskflow. The project is open-source and more details can be found in [10].

\section{CPP-TASKFLOW}

In Cpp-Taskflow, the programming is centered around two classes: tf::Taskflow and tf::Executor. We will explain how to use them in this section.

\subsection{Task Dependency Graph}

In Cpp-Taskflow, a task is a $\mathrm{C}++$ object of Callable type [7]. To create tasks, the first step is to create an object of the tf::Taskflow class. A taskflow object allows you to build a task dependency graph where nodes are tasks and directed edges indicate dependency. Listing 1 shows an example of adding three tasks via the 
emplace method. The emplace method can create multiple tasks at one time. After tasks are created, users can assign names to tasks and specify the dependency between tasks via the name and precede method, respectively. A task A precedes a task B if task B can only run after task A completes its execution.

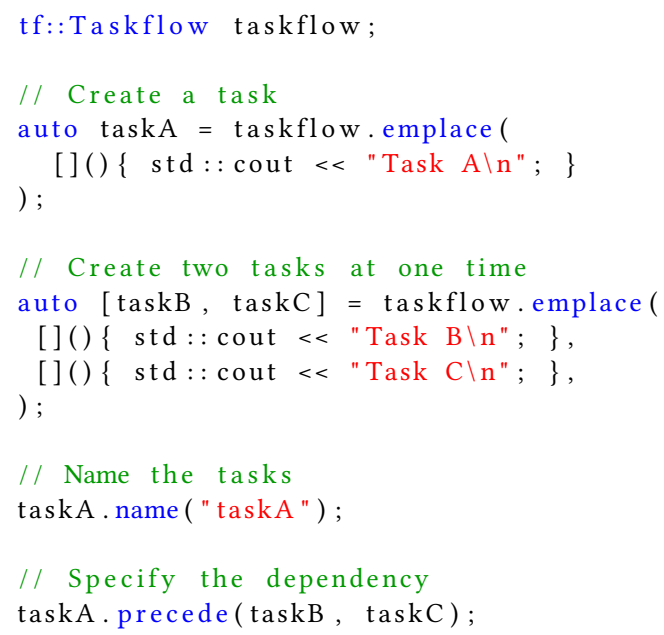

\section{Listing 1: Create a task dependency graph.}

\subsection{Dynamic Tasking}

Cpp-Taskflow has another powerful feature: dynamic tasking that enables a task to create and dispatch a task dependency graph at runtime to obtain dynamic parallelism. Listing 2 shows an example of dynamic tasking. In this example task B spawns a task dependency graph that has three tasks. A task that requires dynamic parallelism has to take an additional argument of type tf::Subflow and uses the emplace method to create a new task dependency graph. The new task dependency graph will by default join its parent task. However, users can make it run independently by calling the detach method. A detached task dependency graph will join the end of its parent's task dependency graph. Figure 1 shows the spawned task dependency graphs in joined and detached modes, respectively. Dynamic tasking empowers users to parallelize frequently used computing patterns such as recursive and nested flows.

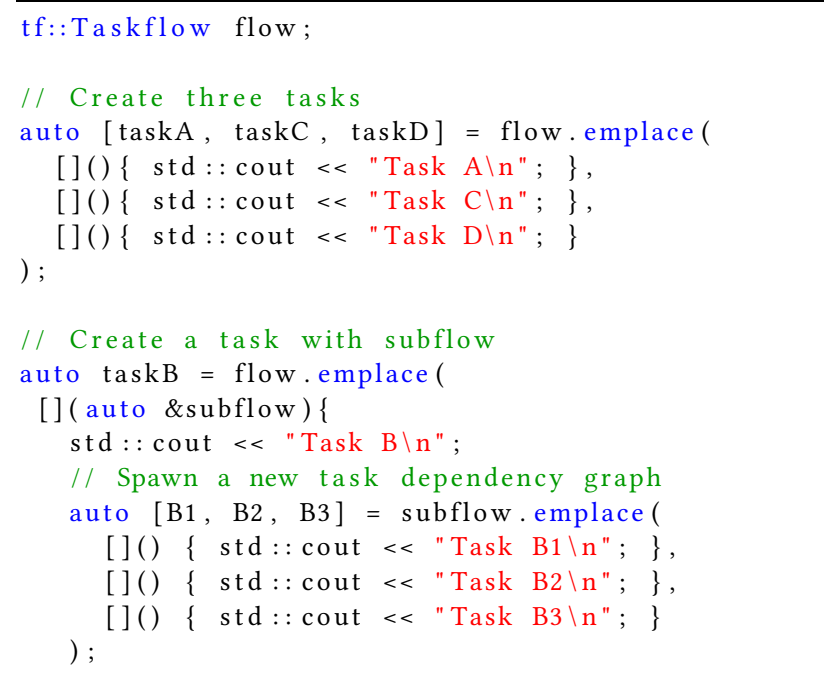

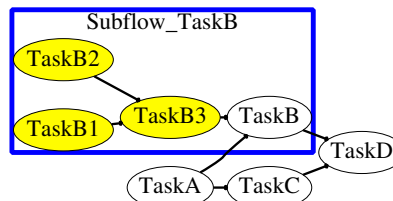

(a) A joined subflow.

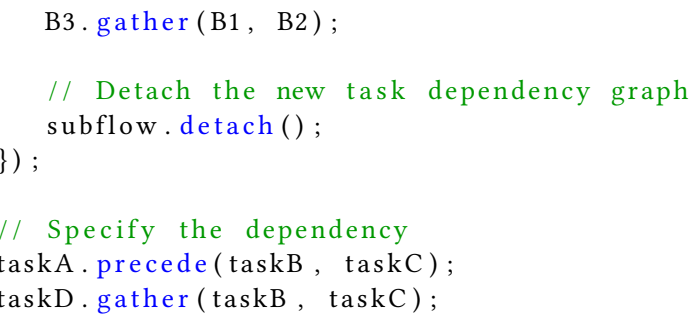

Listing 2: An example of dynamic tasking.

Figure 1: Comparison of joined and detached subflows.

\subsection{Composition}

An useful feature of task dependency graph is the composability. Users can use the composed_of method to compose several task dependency graphs to a large and complex task dependency graph. The composed_of method returns a module task. Users can use the precede method to add dependency between module tasks and other tasks. Listing 3 shows an example of task dependency graph composition.

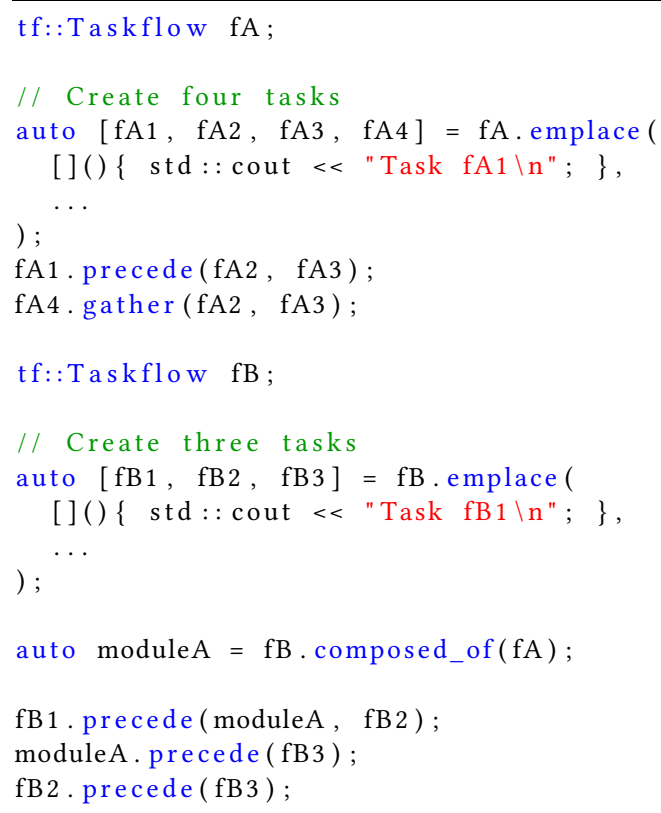

Listing 3: An example of task dependency graph composition. 


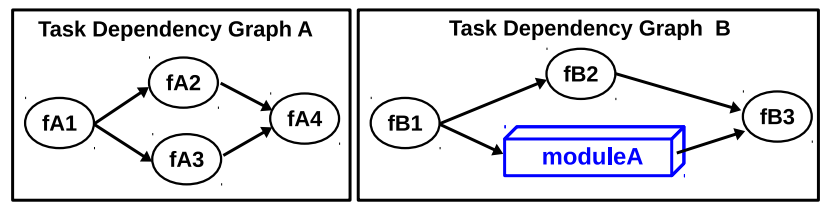

Figure 2: The task dependency graphs of the example in Listing 3.

\subsection{Execution}

After creating task dependency graphs, the next step is to dispatch graphs to an executor object of type tf::Executor. An executor object manages thread construction and destruction and provides several methods to execute task dependency graphs through an efficient work-stealing algorithm. Table 1 summarizes the execution methods and Listing 4 demonstrates the usage of those execution methods.

\begin{tabular}{|c|c|}
\hline Method & Description \\
\hline run & Execute a graph once \\
\hline run_n & Execute a graph multiple times \\
\hline run_until & Execute a graph until a condition is met \\
\hline wait_for_all & Wait until all running graphs finish \\
\hline
\end{tabular}

Table 1: Summary of execution methods.

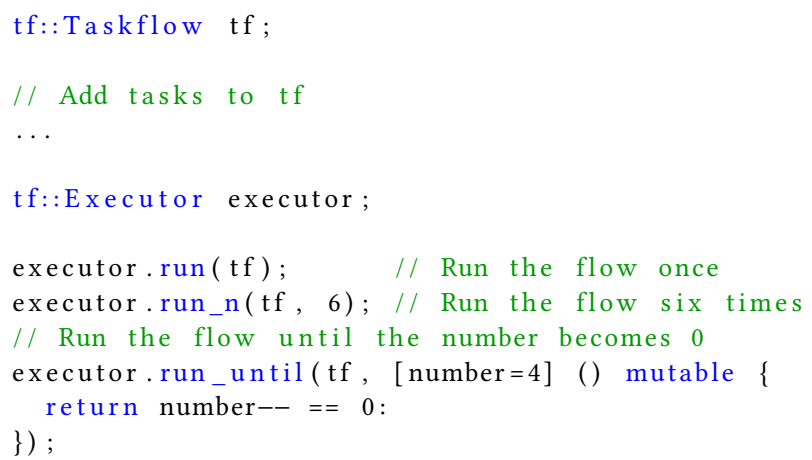

Listing 4: Demonstration of different execution methods.

\subsection{Debugging and Profiling}

Debugging a parallel program is very challenging due to the nondeterministic nature. Cpp-Taskflow supports the visualization of task dependency graphs to let users inspect the task execution flow. Users can use the name method to assign a taskflow object a name and the dump method to export the object's task graph in DOT format [11]. Listing 5 shows an example of naming and dumping a task dependency graph. Figure 3 demonstrates the task dependency graphs of two taskflow objects.

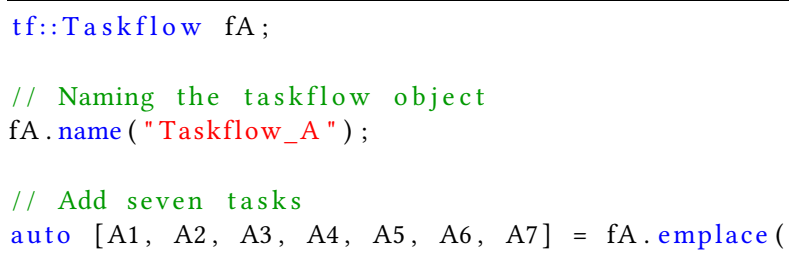

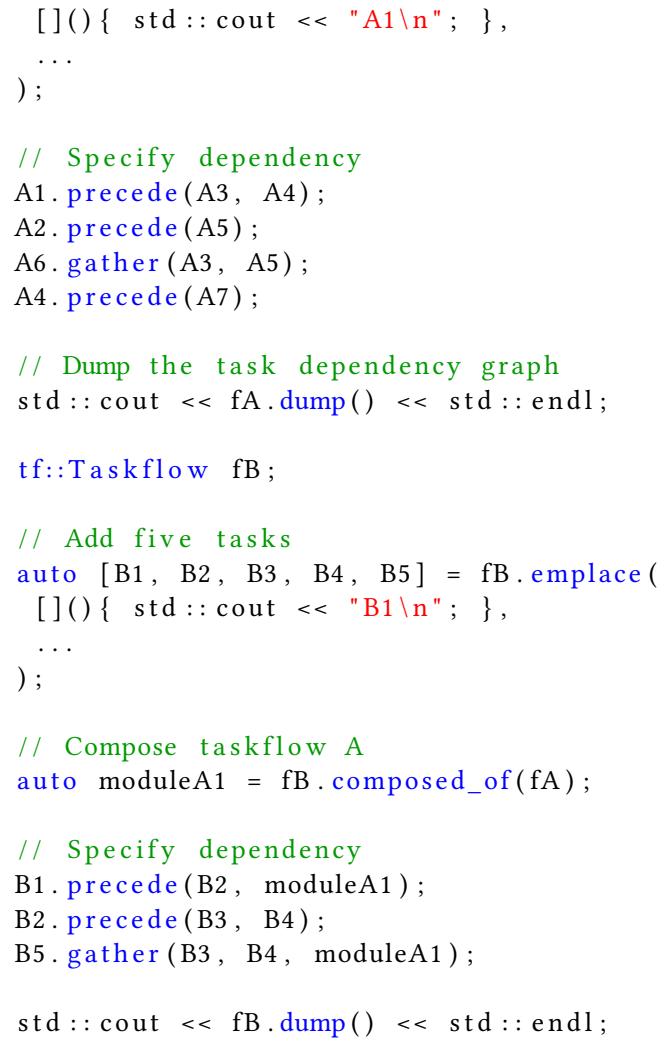

Listing 5: Visualization of a task dependency graph.
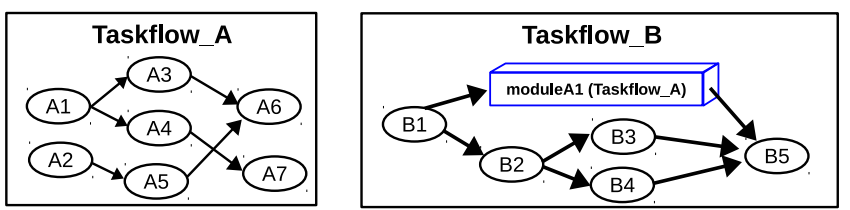

Figure 3: The task dependency graphs of two taskflow objects in Listing 5.

Profiling is very important when developers analyze their application's performance. Cpp-Taskflow allows users to record the thread activities and visualize them in a Chrome browser. To enable profiling, users create an observer of type tf::Executor Observer through executor's make_observer method. An observer will record each task's start time (via on_entry method) and end time (via on_exit method) during execution. The observer can dump the recorded timestamps into a JSON file and users can visualize the execution timeline by loading the JSON file in the chrome://tracing developer tool. Listing 6 shows how to create an observer to monitor the thread activities. Figure 4 displays the task execution timeline in a Chrome browser.

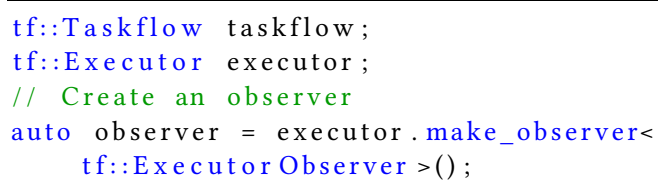




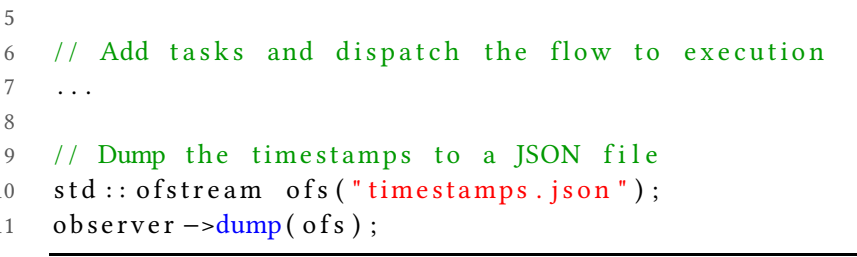

Listing 6: Use an observer to monitor the thread activities.

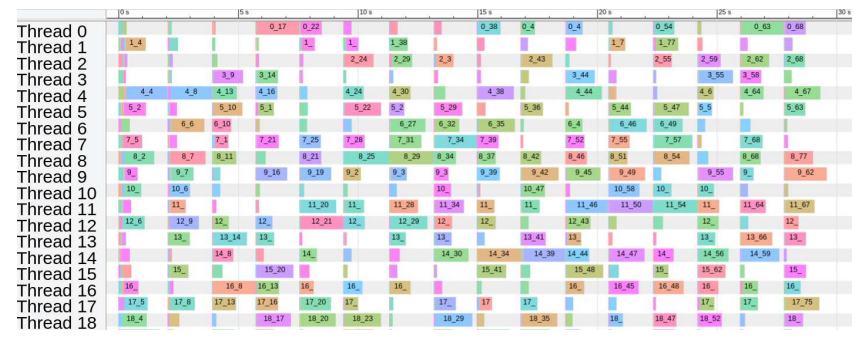

Figure 4: Thread activities displayed in chrome://tracing.

\section{A MACHINE LEARNING APPLICATION}

Machine learning has been successfully applied to several multimedia topics such as image classification, speech recognition and so on [4] [5]. We demonstrate applying Cpp-Taskflow to parallelize a machine learning application: MNIST [12] dataset, and compare its performance and coding effort with OpenMP [9]. The MNIST dataset contains images of handwritten digits and it is widely used to test the effectiveness of machine learning algorithms. In this demonstration, we build a 5-layer deep neural network (DNN) to classify those images. We adopt the task pipeline strategy proposed by [7] to parallelize the DNN training. Each batch starts with a task for forward propagation and then followed by a sequence of gradient calculation and weight update tasks for each layer. We pipeline the gradient calculation and weight update tasks between successive layers to enable parallelism within each batch. Next we create tasks for data shuffle per epoch. We allocate additional data storages to have a shuffle task start earlier preparing the data for later epochs. We compare the implementations of OpenMP [9] and Cpp-Taskflow. OpenMP is the most popular parallel programming library in high-performance computing and we use OpenMP's task depend clause to implement this parallelization strategy. For CppTaskflow, we implement this application using the taskflow object and use the executor's run method for execution. We run all implementations on a machine with a Intel Xeon W-2175 processor and $128 \mathrm{~GB}$ memory and we launch 10 threads in this experiment. The operating system is Ubuntu 18.04 and the OpenMP version is 4.5 (201511). The learning rate is set to 0.0001 and for each number of epochs we run five times and take the average. During the experiment we use the system command taskset to bond the process to the first 10 cores. Figure 5 plots the runtime of both implementations and Table 2 shows the code complexity measured by Lizard [13] ${ }^{1}$. The implementation of Cpp-Taskflow is slightly faster than OpenMP. Regarding the code complexity, OpenMP is $35 \%$ longer than Cpp-Taskflow in lines of code.

${ }^{1}$ Because Lizard takes compiler directives (starts with \#) as a comment, we remove the \# when measuring the OpenMP implementation.

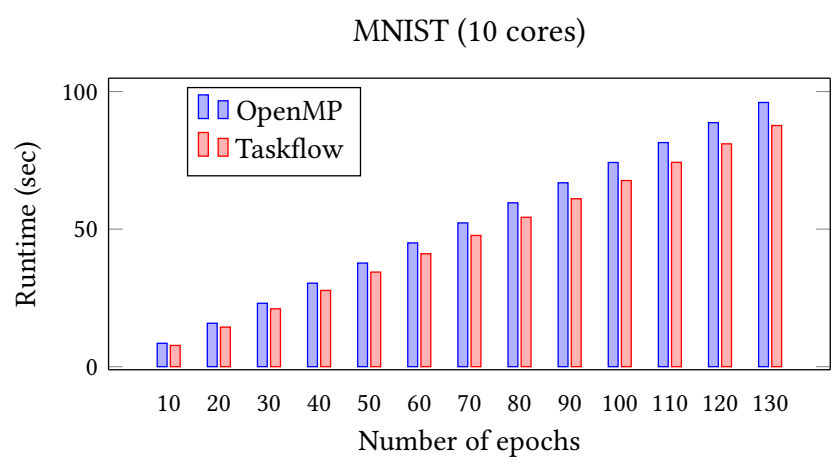

Figure 5: DNN training runtime of OpenMP and CppTaskflow with using taskflow object.

Table 2: Code complexity [13] of the three implementations.

\begin{tabular}{|c|c|c|c|}
\hline Library & Total NLOC & Avg Token & Avg CCN \\
\hline OpenMP & 93 & 1058 & 11 \\
\hline Taskflow & 60 & 600 & 11 \\
\hline
\end{tabular}

NLOC: lines of code. $\mathbf{C C N}$ : cyclomatic complexity number.

\section{AVAILABILITY}

Cpp-Taskflow is open-source on Github [10] under MIT license. The API documentation, tutorials and cookbook are also available on Github. We have presented Cpp-Taskflow at CppCon which is the premier $\mathrm{C}++$ developer conference and the video is on YouTube [14].

\section{ACKNOWLEDGEMENT}

Cpp-Taskflow is supported by NSF Grant CCF-1718883 and DARPA Grant FA 8650-18-2-7843.

\section{REFERENCES}

[1] W. Zhu, P. Cui, Z. Wang, and G. Hua. Multimedia big data computing. IEEE MultiMedia, 22(3):96-c3, July 2015.

[2] Z. Wang, S. Mao, L. Yang, and P. Tang. A survey of multimedia big data. China Communications, 15(1):155-176, Jan 2018.

[3] Samira Pouyanfar, Yimin Yang, Shu-Ching Chen, Mei-Ling Shyu, and S. S. Iyengar. Multimedia big data analytics: A survey. ACM Comput. Surv., 51(1):10:110:34, January 2018.

[4] Jitao Sang, Jun Yu, Ramesh Jain, Rainer Lienhart, Peng Cui, and Jiashi Feng. Deep learning for multimedia: Science or technology? In Proceedings of the 26th ACM International Conference on Multimedia, MM '18, pages 1354-1355, New York, NY, USA, 2018. ACM.

[5] Y. Yan, M. Chen, M. Shyu, and S. Chen. Deep learning for imbalanced multimedia data classification. In 2015 IEEE International Symposium on Multimedia (ISM), pages 483-488, Dec 2015

[6] T.-W Huang and Martin D. F. Wong. OpenTimer: A high-performance timing analysis tool. In IEEE/ACM ICCAD, pages 895-902, 2015.

[7] T.-W Huang, C.-X. Lin, Guannan Guo, and Martin D. F. Wong. Cpp-Taskflow: Fast Task-based Parallel Programming using Modern $\mathrm{C}++$. IEEE IPDPS, pages 974-983, 2019.

[8] Intel Threading Building Blocks. [Online]. Available: https://www.threadingbuildingblocks.org.

[9] OpenMP. [Online]. Available: https://www.openmp.org/.

[10] Cpp-Taskflow. [Online]. Available: https://github.com/cpp-taskflow/cpp-taskflow.

[11] The DOT Language. [Online]. Available: https://www.graphviz.org/.

[12] MNIST. [Online]. Available: http://yann.lecun.com/exdb/mnist/.

[13] Lizard. [Online]. Available: http://www.lizard.ws/

[14] Cpp-Taskflow lightning talk. [Online]. Available: https://www.youtube.com/watch?v=ho9bqIJkvkc. 